\title{
Cierre espontáneo de fístula residual post cierre quirúrgico de fístula aorto-ventricular derecha traumática. Caso clínico
}

ROBERTO GONZÁLEZ L. ${ }^{1}$, CARMEN SANTANDER A. ${ }^{1}$, ENRIQUE SEGUEL S. ${ }^{1}$, ALECK STOCKINS L. ${ }^{1}$, RODRIGO ÁLVAREZ L. ${ }^{2}$, PATRICIO CÁRDENAS M. ${ }^{3}$, RODRIGO CAMPOS M. ${ }^{1}$, EMILIO ALARCÓN C. ${ }^{1}$

\section{Spontaneous closure of a residual fistula after surgical closure of a traumatic aorto-right ventricular fistula. Report of one case}

We report a 16-year-old boy, who suffered a right ventricle penetrating injury caused by a sharp blade, that evolved to cardiac tamponade. He underwent surgery and was discharged four days later. Thirteen days later, a cardiac murmur was found. An echocardiography showed an aorto-right ventricular fistula. Surgical closure was performed through an aortotomy on cardiopulmonary by pass. The control echocardiography showed a small residual fistula, which closed spontaneously three months later.

(Rev Med Chile 2010; 138: 213-216).

Key words: Cardiac tamponade; Cardiopulmonary bypass.

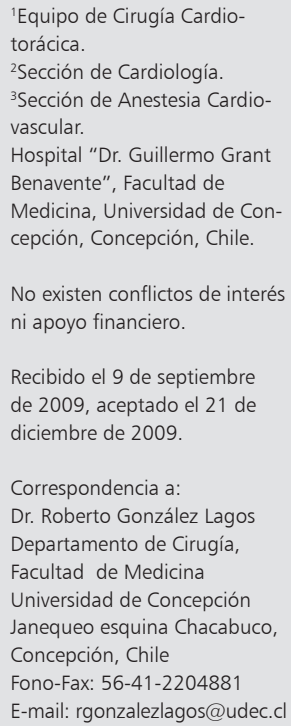

L as lesiones intracardiacas post traumáticas son infrecuentes ${ }^{1,2}$. En el caso particular de la fístula aorto-ventricular derecha traumática, desde 1958 a la fecha, sólo se han comunicado en la literatura $44 \operatorname{casos}^{3-6}$. El cierre quirúrgico de la fístula es considerado el tratamiento de elección, con excelentes resultados. No encontramos comunicaciones de una fístula residual post cierre quirúrgico, que haya evolucionado con cierre espontáneo. Presentamos un caso de fístula aortoventricular derecha traumática en que se realizó el cierre quirúrgico y se demostró una fístula residual: ésta se manejó en forma conservadora y cerró espontáneamente.

\section{Caso Clínico}

Hombre de 16 años, sin antecedentes mórbidos de importancia. Recibió una herida con arma blanca paraesternal en el tercer espacio intercostal izquierdo. Evolucionó con taponamiento cardíaco y se realizó una esternotomía exploradora. Se encontró un hemopericardio y una herida cardíaca de aproximadamente 10 milímetros de longitud en relación con el tracto de salida del ventrículo derecho. Se suturó con polipropileno 3-0 punto continuo. No se encontraron otras lesiones. El paciente evolucionó favorablemente en el postoperatorio inmediato, asintomático y sin soplo; fue dado de alta a su domicilio al cuarto día postoperatorio. En el control, al decimotercer día, se encontró un frémito precordial y se auscultó un soplo continuo en el mesocardio de intensidad 4/6. El ecocardiograma transesofágico reveló a 5 milímetros sobre el velo coronariano derecho un flujo turbulento que comunicaba la raíz aórtica con el tracto de salida del ventrículo derecho, con un gradiente máximo de $90 \mathrm{mmHg}$, compatible con una fístula aorto-ventricular derecha (Figura 1). Se decidió el cierre quirúrgico, el cual se rea- 
lizó el decimonoveno día posterior a la primera intervención.

Procedimiento quirúrgico: Se abordó por esternotomía media vertical y se liberaron las adherencias. Se encontró un frémito en el tracto de salida del ventrículo derecho y se calculó a través de gasometría un Qp:Qs = 1,5:1. Empleando circulación extracorpórea a normotermia, con canu- lación arterial en aorta ascendente y canulación venosa única, se detuvo el corazón con cardioplejia cristaloide anterógrada. La fístula se expuso por aortotomía. Se encontró a 5 milímetros sobre el plano valvular y a 2 milímetros a la izquierda del ostium coronario derecho una lesión lineal de 5 milímetros con un "flap" de disección en su borde superior (Figura 2). La lesión se reparó y se fijó el "flap" con puntos separados de polipropileno

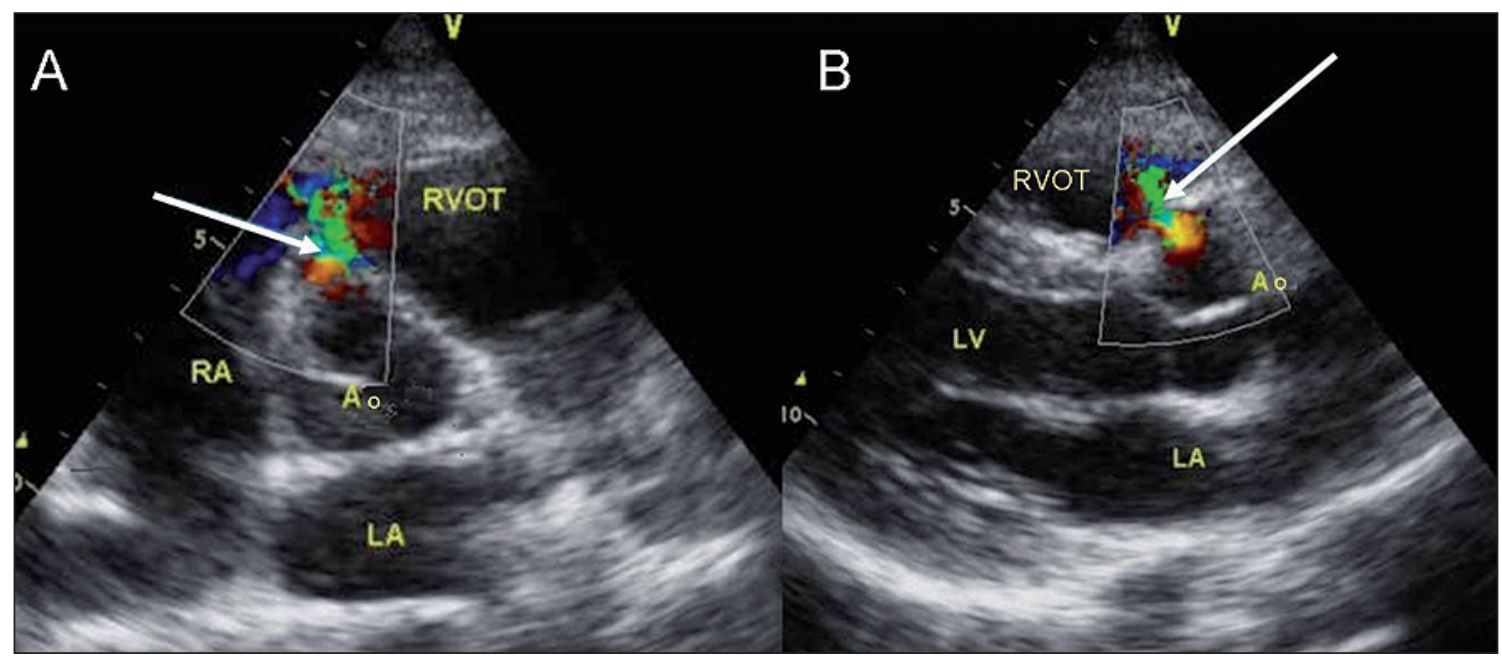

Figura 1. A y B. Ecocardiograma transtorácico muestra fístula aorto-ventricular derecha traumática (flecha) (Ao: aorta; LV: ventrículo izquierdo; LA aurícula izquierda; RA: aurícula derecha; RVOT: tracto de salida de ventrículo derecho).

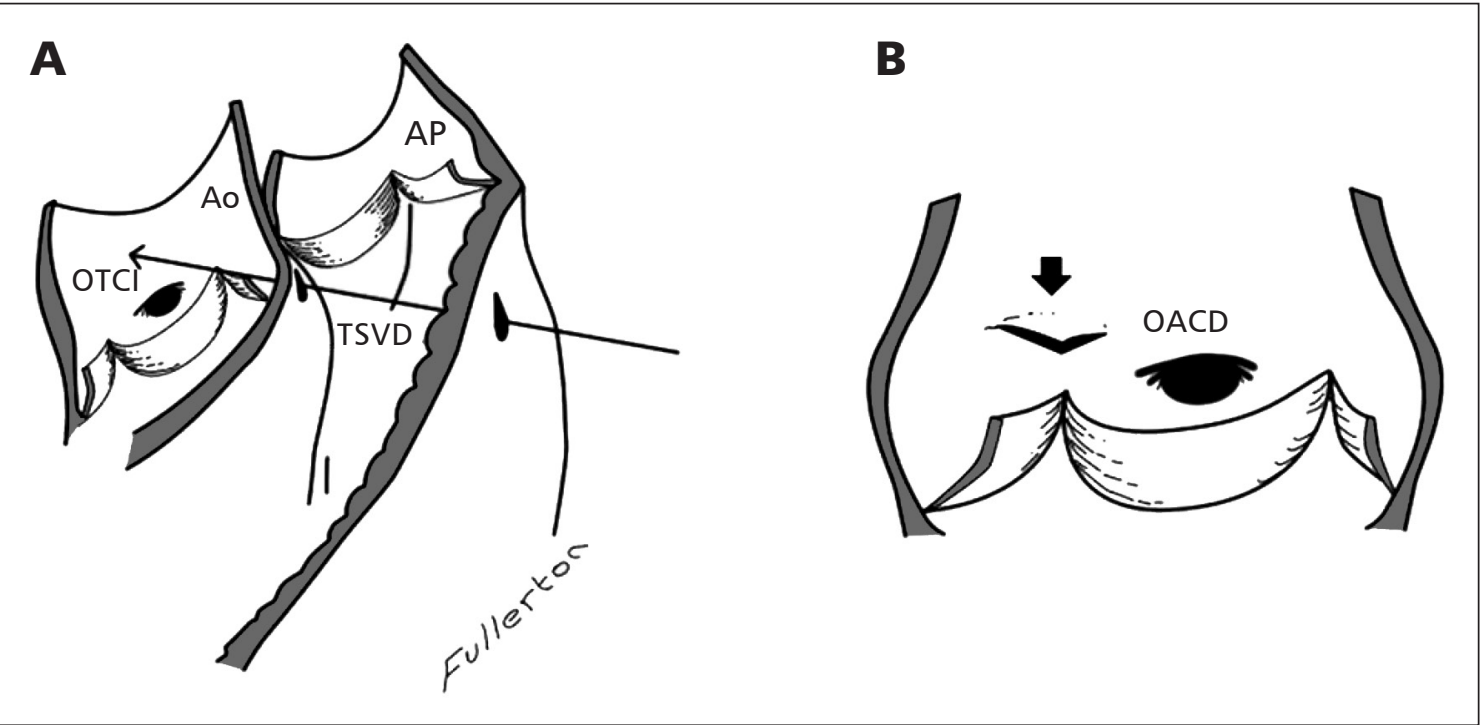

Figura 2. A. Esquema que muestra el trayecto de la herida penetrante (flecha). B. Relación entre la lesión (flecha), el ostium coronario derecho y la válvula aórtica. (Ao: aorta; AP: arteria pulmonar; OTCl: ostium tronco coronario izquierdo; OACD: ostium arteria coronaria derecha; TSVD: tracto de salida de ventrículo derecho). 
5-0. Posterior a la salida de circulación extra corpórea se comprobó la desaparición del frémito y se calculó nuevamente el Qp:Qs con desaparición del shunt.

Evolución postoperatoria: El paciente evolucionó en el postoperatorio inmediato sin incidentes. El ecocardiograma transtorácico al cuarto día postoperatorio demostró una pequeña fístula residual de aorta a ventrículo derecho, en la zona donde se encontraba la lesión. Se decidió manejo expectante y se dio de alta hospitalaria al cuarto día postoperatorio, asintomático y sin soplo. A los 3 meses postoperado, el ecocardiograma transtorácico estaba dentro de límites normales, sin dilatación de cavidades derechas y sin evidencias de fístula residual. A los 18 meses el paciente se encuentra en capacidad funcional I, asintomático, sin soplo y el ecocardiograma transtorácico no muestra hallazgos significativos.

\section{Discusión}

Son pocos los casos comunicados de fístula aorto-ventricular derecha traumática post herida penetrante cardíaca, entre ellos, una serie presentada por el equipo de la Pontificia Universidad Católica de Chile ${ }^{3}$.

En la revisión de Sammuels y cols ${ }^{4}$, en que se describen 40 casos, la mayoría de los traumatismos son penetrantes, en hombres jóvenes (edad promedio 28, 3 años). El tiempo desde el traumatismo al diagnóstico de la fístula es variable, incluso puedeproducirse varios años después del traumatismo. Las manifestaciones clínicas van desde pacientes asintomáticos hasta la insuficiencia cardíaca descompensada. La presencia de soplo continuo está descrita en la mayoría de los pacientes; este soplo puede ser detectado incluso varios días después del traumatismo. La mayoría de las veces estas lesiones son aisladas, sin embargo, se pueden acompañar de otras lesiones intra cardíacas, como insuficiencia aórtica, defectos septales (comunicación inter auricular y comunicación inter ventricular) o de insuficiencia mitral ${ }^{4}$.

El diagnóstico de la fístula aorto-ventricular derecha actualmente se basa en la sospecha clínica y se confirma con la ecocardiografía ${ }^{7}$. El tratamiento recomendado es el cierre quirúrgico, ya que según lo descrito no evoluciona con cierre espontáneo y su evolución natural sería hacia la insuficiencia cardíaca congestiva ${ }^{3-6}$. Todos los casos tratados quirúrgicamente en la literatura lo fueron bajo circulación extra corpórea y se abordaron a través de aortotomía, ventriculotomía derecha o ambas. En la mayoría de los casos el cierre de la fístula fue directo, con puntos apoyados en teflón ${ }^{3-6}$.

Se han desarrollado alternativas de cierre percutáneo para diversas fístulas y comunicaciones intra cardíacas. No encontramos comunicaciones en que se realizara cierre no quirúrgico para este tipo de lesión post traumática.

En el caso presentado, el diagnóstico se sospechó por la aparición de un soplo continuo en el control a los trece días post operado del taponamiento agudo, esto fue confirmado con un ecocardiograma transtorácico y luego con un ecocardiograma transesofágico.

La lesión se abordó por aortotomía y se realizó el cierre directo. Una vez fuera de circulación extra corpórea, se calculó el Qp:Qs por gasometría, que demostró la desaparición del shunt. Además, fue evidente la desaparición del frémito y con el tórax cerrado, a la auscultación desapareció el soplo. Ya que no se disponía de ecocardiograma transesofágico intraoperatorio, y por lo anteriormente descrito, se consideró satisfactoria la reparación. Sin embargo, en el ecocardiograma transtorácico de control al cuarto día postoperado se demostró una filtración residual en la zona de la reparación, ante lo cual se decidió manejo expectante.

El paciente se mantuvo asintomático y en el control a los 3 meses postoperado se demostró con un ecocardiograma transtorácico el cierre espontáneo de la fístula residual descrita.

No encontramos comunicaciones de filtración residual post cierre de fístula aorto-ventricular derecha traumática que evolucionara con cierre espontáneo. Dada la evolución clínica de nuestro paciente creemos que en el caso de presentarse filtraciones residuales post cierre quirúrgico, puede considerarse una conducta expectante.

Agradecimientos: Al Dr. Demian Fullerton M., por la confección de la figura quirúrgica.

\section{Referencias}

1. Meredith JW, Hoth JJ. Thoracic trauma: when and how to intervene. Surg Clin North Am 2007; 87: 95-118.

2. González R, Zalaquett R, Chamorro G, Lema G. [Massi- 
Cierre espontáneo de fístula residual post cierre quirúrgico de fístula aorto-ventricular - R. González L. et al

ve tricuspid valve insufficiency after blunt chest trauma: report of one case]. Rev Med Chile 2008; 136: 1034-8.

3. Moran S, Maturana G, Urzúa J, Franck R, Dubernet J. Elective correction of intracardiac lesions resulting from penetrating wounds of the heart. Thorax 1979; 34: 45963.

4. Samuels LE, Kaufman MS, Rodriguez -Vega J, Morris RJ, Brockman SK. Diagnosis and management of traumatic aorto-right ventricular fistulas. Ann Thorac Surg 1988; 65: 288-92.
5. Kaya A, Dekkers P, Loforte A, Jaarsma W, Morshuis WJ. Traumatic aorto-right ventricular fistula with aortic insufficiency. Ann Thorac Surg 2005; 80: 2362-4.

6. Cakir C, Duygu H, Kilicaslan B, Ertas F, Ozen N, Nazli C, Ergene O. Postoperative diagnosis of aorto-right ventricular outflow tract fistula caused by stab wound: a case report. J Am Soc Echocardiogr 2007; 20: 1415.e5-7.

7. Walpot J, Klazen C, Hokken R, Sorgedragerj, Hoevenaar $\mathrm{M}$, Den Brader J. Aorto-right ventricular fistula as an occasional finding. Eur J Echocardiogr 2005; 6: 65-6. 